\title{
The Senior Toronto Oncology Panel (STOP) Study: Research Participation for Older Adults With Cancer and Caregivers
}

\author{
Martine T.E. Puts, RN, PhDa; Schroder Sattar, RN, MN; Takami Fossat, RNª Margaret I. Fitch, RN, PhD; \\ Geraldine J. Macdonald, RN, PhD; ; Tina Hsu, MD' Ewa Szumacher, MD, MEdc; \\ Douglas A. Stephens, BASc, MBA*; Joseph Robinson, BA, BEd*; David Macdonald*; Andrew S. Choate*; \\ Eric Pitters*; Barbara Liu, MD; Lianne Jeffs, RN, PhD; ${ }^{\text {e; }}$ Katherine S. McGilton, RN, PhD ${ }^{\text {a,f; }}$ \\ and Shabbir M.H. Alibhai, MD, MSc ${ }^{g}$
}

\begin{abstract}
Background: Patient engagement in research may lead to better-designed studies and improved health outcomes. The objectives of this study were to identify the research priorities of older adults with cancer (OAWCs) and their caregivers and examine how to engage these individuals in research teams and what supports are needed. Methods: We conducted 3 public meetings and 7 focus groups to delineate research priorities and the supports needed to facilitate integration of OAWCs and their caregivers on research teams. Results: A total of 33 older adults and 19 caregivers attended a public meeting and 27 older adults and 17 caregivers participated in a focus group. Most of the OAWCs and their caregivers had never participated in research before. Three themes were identified from the focus groups: (1) motivation to be on a team; (2) ability to make meaningful contributions; and (3) logistical considerations to facilitate engagement. Most participants were motivated to be a research team member and be involved in all steps of research if it could benefit them or future patients and caregivers. OAWCs and their caregivers were highly motivated to improve outcomes. Required logistics included flexibility regarding time and location, accessibility to computer technology, transportation support, materials worded in lay language, and attending/having short training sessions, as well as the presence of peer support. Conclusions: OAWCs and their caregivers are very motivated and willing to participate in research and to be research team members. Logistics and the social aspects of being on a team are important.
\end{abstract}

J Natl Compr Canc Netw 2017;15(10):1208-1215 doi: 10.6004/jnccn.2017.0159

Older adults are underrepresented in clinical cancer research. ${ }^{1-7}$ Evidence shows that engaging patients in the research process can lead to increased enrollment and retention rates ${ }^{8-11}$ and enhanced credibility of the

From a Lawrence S. Bloomberg Faculty of Nursing, University of Toronto, Toronto; 'Department of Medical Oncology, Ottawa Hospital Cancer Centre, Ottawa; 'Odette Cancer Centre, Sunnybrook Health Sciences

Centre, Toronto; ${ }^{d}$ Regional Geriatric Program of Toronto, Sunnybrook

Health Sciences Centre, University of Toronto, Toronto; 'Keenan Research

Centre of Li Ka Shing Knowledge Institute, St. Michael's Hospital, Toronto;

'Toronto Rehabilitation Institute, University Health Network, Toronto;

gDepartment of Medicine and Institute of Health Policy, Management, and

Evaluation, University Health Network and University of Toronto, Toronto,

Ontario, Canada.

*Patient representatives.

Submitted February 23, 2017; accepted for publication May 19, 2017.

Dr. Hsu has disclosed that she has served on an advisory board for Pfizer and Celgene (unrelated to this project). The remaining authors have disclosed that they have no financial interests, arrangements, affiliations, or commercial interests with the manufacturers of any products discussed in this article or their competitors. findings, which in turn increases translation of research into clinical practice. $^{12}$ Patient engagement in research is expected to lead to improved health outcomes and better uptake of findings because the research is

Dr. Puts is supported by a Canadian Institutes of Health Research New Investigator Award. Funding for this study was provided by Canadian Institutes of Health Research program: SPOR Patient Engagement - Collaboration (grant number 344008), and St. Michael's Hospital, University Health Network, Odette Cancer Centre, Leukemia \& Lymphoma Society Canada, and Prostate Cancer Canada.

A poster presentation was presented at the International Society of Geriatric Oncology 2016 Annual Conference; November 17-19, 2016; Milan, Italy and the annual meeting of the MASCC/ISOO 2016 Annual Meeting on Supportive Care in Cancer; June 23-25, 2016; Adelaide, Australia. An oral presentation was given at the International Society of Geriatric Oncology 2015 Annual Conference; November 12-14, 2015; Prague, Czech Republic.

Correspondence: Martine T.E. Puts, RN, PhD, Lawrence S. Bloomberg Faculty of Nursing, University of Toronto, 155 College Street, Suite 130 , Toronto, ON M5T 1P8 Canada. E-mail: Martine.Puts@utoronto.ca 
expected to be more relevant for patients. ${ }^{13}$ Stakeholder participation in determining crucial areas of research and healthcare services has been recognized in the United Kingdom by the British National Institute of Health since 1996, leading to the project INVOLVE. ${ }^{14}$ Additionally, the US Patient Centered Outcomes Research Institute (PCORI; established in 2010) strongly emphasizes the importance of stakeholders and patient engagement in the research process. ${ }^{15,16}$

There is also a movement in Canada to engage patients in research. Patient engagement has been defined by the Canadian Institutes of Health Research as "meaningful and active collaboration in governance, priority setting, conducting research and knowledge translation. Depending on the context, patient-oriented research may also engage people who bring the collective voice of specific, affected communities." ${ }^{13}$

Despite the increasing requirements of funding agencies to engage patients on research teams, there are few data to suggest the most effective approach. ${ }^{8}$ Additionally, there is a paucity of research on what older adults with cancer (OAWCs) see as research priorities for cancer, how they feel about being research team members, and what supports and resources they require to participate in this process; this is important to examine because engagement can lead to patient frustration. ${ }^{17}$
The goals of this study were to understand the research priorities of OAWCs and their caregivers, and examine how OAWCs and their caregivers can be engaged in research teams and what supports are needed.

\section{Methods}

\section{Study Design}

This study was designed with OAWCs and caregiver representatives on the team and was conducted in 2 phases (Figure 1): public meetings followed by focus groups. At the end of each phase, participants were invited to join the participant panel aimed at engaging them to be on a future research team.

The study was approved by the Research Ethics Board of the University of Toronto. All participants provided consent before participating in the surveys and focus groups.

\section{Participant Recruitment}

Partners for this project included hospitals in the region, health charities, and advocacy organizations for patients and older adults. For the public meetings and focus groups, older adults aged $\geq 60$ years who had been diagnosed with any type of cancer in the past 10 years and their caregivers were recruited separately using several strategies, including study Web site; recruitment posters and business cards in oncology waiting areas in partner hospitals and senior

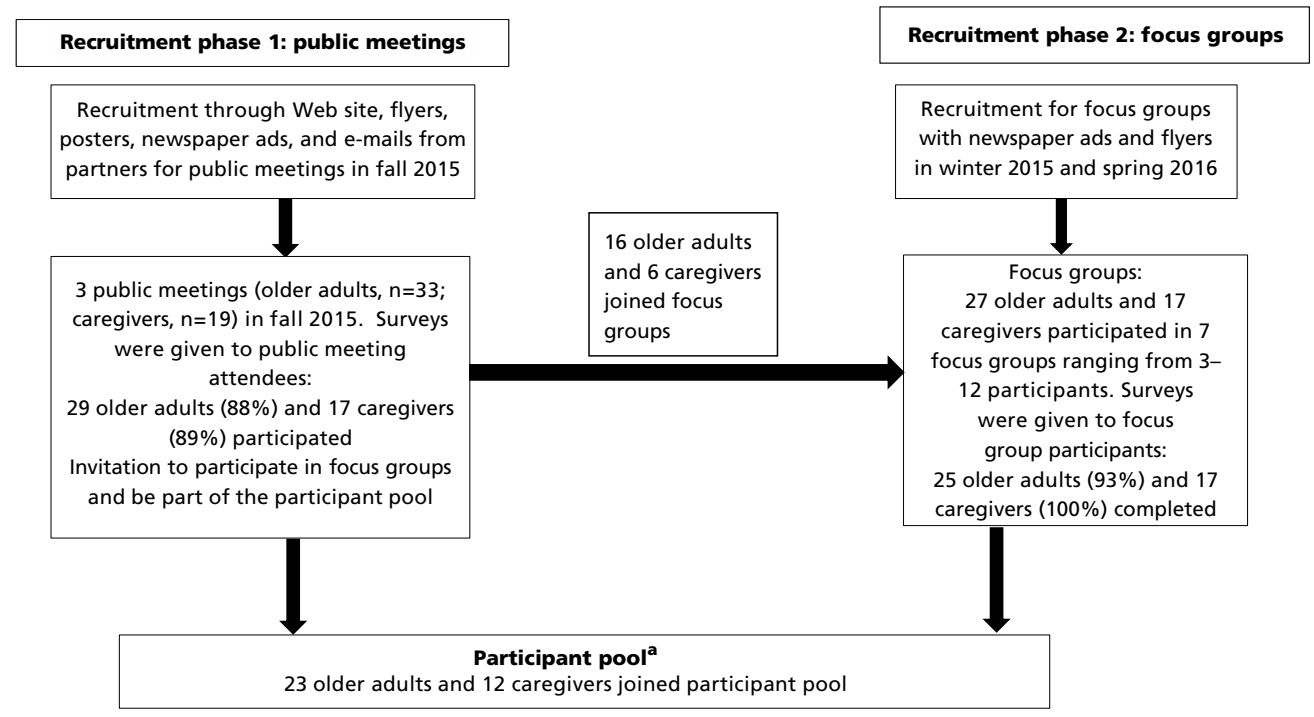

Figure 1. Study timeline and participant flow through the phases of the study. aDue to anonymity in the survey, as well as a few incomplete surveys, we could not determine precisely how many participants in the participant pool came from phase 1, phase 2, or both. 
community centers; advertisements in local newspapers in the Greater Toronto Area (GTA), Kijiji, and Craigslist, and on the Volunteer Toronto Web site; and advertisements through our partner organizations (via newsletters and e-mails).

Attendees who needed transportation arrangements were provided with taxi services to and from the venue for both public meetings and focus groups, and lunch was provided.

\section{Public Meetings}

Three public meetings were held to better understand the research priorities of OAWCs and caregivers. The public meetings took place on Saturdays at 3 different University of Toronto locations in September, October, and November of 2015 and lasted for 3 hours, with half an hour for a reception and farewell. Research priorities were identified in small group discussions and notes were taken by nursing students. The research priorities from the public meetings were summarized.

Surveys were administered at the end of the public meetings to measure satisfaction, understand how to improve future meetings, and invite OAWCs and caregivers to participate in a focus group and join the participant pool. The survey collected information on age, sex, education level, cancer diagnosis, years since diagnosis, and self-rated health. Completion of the survey was voluntary.

\section{Focus Groups}

Focus groups aimed to explore what OAWCs and caregivers felt about being co-researchers on the team and to identify supports needed to enable/ facilitate older adults and caregiver participation as research team members. The 7 focus groups were held in public libraries across the GTA from December 2015 through April 2016 and lasted 2 hours. All focus groups included both OAWCs and their caregivers in the same group, and were moderated by an experienced moderator and audiorecorded (a guide with the moderation questions is provided in supplemental eAppendix 1, available with this article at JNCCN.org). A second member of the research team took notes. At the end of the focus groups, participants were asked to complete the survey, which was voluntary. Participants were also invited to join the participant panel. The num- ber of focus groups was determined based on data saturation (eg, no new themes emerged).

\section{Data Analysis}

Survey responses were entered into a Microsoft Excel spreadsheet and descriptive analyses (frequencies, median, and range) were used to describe the characteristics of the participants.

The audio recordings from the focus groups were transcribed verbatim and the content then analyzed, along with the handwritten field notes using thematic content analysis. ${ }^{18-20}$ The team explored and identified emerging themes through reading and coding the focus group transcripts and notes taken by team members. The findings of OAWCs and caregivers were analyzed together because the discussion was held together, and no differences in answers were noted by the team members. A summary of the findings of each focus group was sent to each participant with a prestamped return envelope. Participants could correct the findings, add missing information, and return the modified summary to the research team as a form of member checking.

\section{Results}

\section{Public Meetings}

Table 1 presents a description of the participants. A total of 33 OAWCs and 19 caregivers attended a public meeting. Although 29 OAWCs completed at least part of the survey, only $26(79 \%)$ answered the sociodemographic questions. The median age for OAWCs was 71 years; most $(n=23 ; 79 \%)$ lived at home and one-third $(n=9)$ lived alone. A total of 17 OAWCs $(60 \%)$ rated their health as "good" or "excellent"; 9 (31\%) were receiving some form of active cancer treatment.

The median age of caregivers was 71 years; 11 were a spouse or partner. All respondents had a high school education or higher. The median number of caregiving was 3.5 years (range, 0-21 years), and 13 caregivers $(76 \%)$ considered their health to be "good," "very good," or "excellent."

\section{Research Priorities of OAWCs and Caregivers}

Table 2 lists the research priorities identified by OAWCs and caregivers at the public meetings. The most commonly voiced priorities were finding a cure for the disease and integrating complementary and 
Older Adult Engagement in Research

\begin{tabular}{|c|c|c|c|c|}
\hline \multirow[b]{2}{*}{ Older Adults $(n=29)$} & \multicolumn{2}{|c|}{ Public Meeting Participants } & \multicolumn{2}{|c|}{ Focus Group Participants } \\
\hline & $\begin{array}{l}\text { Older Adults } \\
\quad(n=29)\end{array}$ & $\begin{array}{c}\text { Caregivers } \\
(n=17)\end{array}$ & $\begin{array}{l}\text { Older Adults } \\
\quad(n=25)\end{array}$ & $\begin{array}{c}\text { Caregivers } \\
(n=17)\end{array}$ \\
\hline Median age (range), y & $71(61-89)$ & $71(25-88)$ & $72(55-88)$ & $67(29-80)$ \\
\hline \multicolumn{5}{|l|}{ Education } \\
\hline 9-12 years (high school) & $(n=6) 21 \%$ & $(n=2) 12 \%$ & $(n=12) 48 \%$ & $(n=4) 24 \%$ \\
\hline$\geq 13$ years (post-secondary) & $(n=18) 62 \%$ & $(n=11) 65 \%$ & $(n=13) 52 \%$ & $(n=12) 71 \%$ \\
\hline Prefer not to say & $(n=5) 17 \%$ & $(n=2) 12 \%$ & & $(n=1) 6 \%$ \\
\hline Living at home & $(n=23) 79 \%$ & $(n=16) 94 \%$ & $(n=24) 96 \%$ & $(n=16) 94 \%$ \\
\hline Currently living alone & $(n=9) \quad 31 \%$ & $(n=6) 35 \%$ & $(n=7) \quad 28 \%$ & $(n=4) 24 \%$ \\
\hline \multicolumn{5}{|l|}{ Comorbidities $^{\mathrm{a}}$} \\
\hline Median number (range) & $1(0-6)$ & $0(0-6)$ & $1(0-5)$ & $0(0-3)$ \\
\hline Arthritis & $(n=9) 31 \%$ & $(n=4) 24 \%$ & $(n=8) 32 \%$ & $(n=6) 35 \%$ \\
\hline Heart conditions & $(n=6) 21 \%$ & $(n=1) 6 \%$ & $(n=7) 28 \%$ & $(n=2) 12 \%$ \\
\hline Stroke & $(n=1) 3 \%$ & $(n=1) 6 \%$ & & \\
\hline Diabetes & $(n=5) 17 \%$ & $(n=3) 18 \%$ & $(n=3) 12 \%$ & $(n=2) 12 \%$ \\
\hline Hypertension & & $(n=1) 6 \%$ & $(n=2) 8 \%$ & $(n=1) 6 \%$ \\
\hline Digestive problems & $(n=5) 17 \%$ & $(n=2) 12 \%$ & & $(n=2) 12 \%$ \\
\hline Asthma & $(n=3) 10 \%$ & $(n=1) 6 \%$ & $(n=1) 4 \%$ & $(n=2) 12 \%$ \\
\hline Kidney problems & $(n=1) 3 \%$ & & & \\
\hline Vision problem & & $(n=1) 6 \%$ & & $(n=2) 12 \%$ \\
\hline Multiple sclerosis & & & & $(n=1) 6 \%$ \\
\hline Brain aneurysm & & $(n=1) 6 \%$ & & $(n=1) 6 \%$ \\
\hline Median years since cancer diagnosis (range) & $3(1-21)$ & N/A & $4(1-21)$ & $\mathrm{N} / \mathrm{A}$ \\
\hline Cancer type & & & b & \\
\hline Prostate & $(n=6) 20 \%$ & \multirow{11}{*}{$\mathrm{N} / \mathrm{A}$} & $(n=4) 17 \%$ & \multirow{11}{*}{$\mathrm{N} / \mathrm{A}$} \\
\hline Colorectal & $(n=3) 10 \%$ & & & \\
\hline Breast & $(n=3) 10 \%$ & & $(n=3) 13 \%$ & \\
\hline Hematologic & $(n=5) 17 \%$ & & $(n=10) 38 \%$ & \\
\hline Gynecologic & $(n=3) 10 \%$ & & $(n=5) 18 \%$ & \\
\hline Head \& neck & $(n=4) 14 \%$ & & $(n=1) 4 \%$ & \\
\hline Kidney \& bladder & $(n=1) 3 \%$ & & $(n=2) 8 \%$ & \\
\hline Pancreatic & $(n=1) 3 \%$ & & $(n=1) 4 \%$ & \\
\hline Melanoma & $(n=1) 3 \%$ & & & \\
\hline Lung & $(n=1) 3 \%$ & & & \\
\hline Liver & $(n=1) 3 \%$ & & & \\
\hline Caregiver who has cancer (caregiver only) & $\mathrm{N} / \mathrm{A}$ & $(n=5) 29 \%$ & N/A & $(n=5) \quad 29 \%$ \\
\hline Self-reported health status good to excellent & $(n=17) 60 \%$ & $(n=13) 76 \%$ & $(n=19) 76 \%$ & $(n=13) 76 \%$ \\
\hline \multicolumn{5}{|l|}{ Types of cancer treatment received } \\
\hline Surgery & $(n=12) 41 \%$ & \multirow{5}{*}{$\mathrm{N} / \mathrm{A}$} & $(n=10) 40 \%$ & \multirow{5}{*}{$\mathrm{N} / \mathrm{A}$} \\
\hline Radiation & $(n=13) 45 \%$ & & $(n=8) 32 \%$ & \\
\hline Chemotherapy & $(n=7) 24 \%$ & & $(n=9) 36 \%$ & \\
\hline Targeted therapy & $(n=3) 10 \%$ & & $(n=4) 16 \%$ & \\
\hline Hormonal & $(n=2) 7 \%$ & & $(n=3) 12 \%$ & \\
\hline Currently on treatment & $(n=9) 31 \%$ & $(n=1) 6 \%$ & $(n=11) 44 \%$ & $(n=0) 0 \%$ \\
\hline Median years of caregiving (range) & N/A & $3(0-21)$ & N/A & $3.5(0-21)$ \\
\hline \multicolumn{5}{|l|}{ Relationship to patient } \\
\hline Spouse/partner & \multirow{6}{*}{ N/A } & $(n=11) 65 \%$ & \multirow{6}{*}{$\mathrm{N} / \mathrm{A}$} & $(n=7) 41 \%$ \\
\hline Child & & $(n=1) 6 \%$ & & $(n=2) 12 \%$ \\
\hline Friend/neighbor & & $(n=2) 12 \%$ & & $(n=3) 18 \%$ \\
\hline In-laws & & $(n=1) 6 \%$ & & $(n=2) 12 \%$ \\
\hline Other & & & & $(n=2) 12 \%$ \\
\hline Prefer not to say & & $(n=2) 12 \%$ & & $(n=1) 6 \%$ \\
\hline
\end{tabular}

Abbreviation: N/A, not applicable.

a May not add up to $100 \%$ due to rounding.

bOne patient was diagnosed with 2 different cancers. 
Puts et al

\section{Table 2. Research Priorities Identified}

\section{- Finding a cure}

- Benefits/harms of alternative and complementary medicine

- Improving communication and information exchange between patient and team

- How to use technology to find best information

- Using technology to prepare videos of patients talking about their experiences for other patients to see when diagnosed and making treatment decisions to provide peer support

- Role of food/nutrients to eat/avoid during and after treatment

- More on treatment side effects that occur in older adults

alternative medicine with traditional therapies to enhance well-being throughout the cancer treatment trajectory.

\section{Satisfaction With Public Meeting}

All respondents were satisfied with the meeting except 1 , and all were satisfied with the length of the meeting except 2 . All but 1 stated that they would attend a future public meeting to learn more about research.

A total of 17 caregivers returned the survey. All said they were satisfied with the meeting; most $(n=14)$ said they would definitely and 3 would possibly attend future meetings on research.

\section{Findings From Focus Groups Discussions}

A total of 27 OAWCs and 17 caregivers participated in 1 of the 7 focus groups (Table 1), from which 3 themes emerged (Table 3): (1) motivation to be on a research team; (2) ability to make meaningful contributions; and (3) logistics required to facilitate engagement. Quotes from OAWCs and caregivers are provided in supplemental eTable 1.

Motivation to Be on a Team: Fewer than half of the OAWCs had participated in any type of study; caregivers had even less exposure to studies. Despite the limited exposure to research but based on their experiences as OAWCs and in the cancer treatment environment, most were very motivated to be on a team to improve care. Most OAWCs and caregivers had never been invited to participate in research. Several indicated that they felt they were not invited to participate in research because they perceived that older adults in Canadian society were not valued, even though they believed older adults could contribute very valuable insights based on their experiences. Some recalled having actively looked for clinical trials, which they saw as valuable for future treatments, but being told they were not eligible to participate. Many OAWCs indicated they did not know how to find clinical trials. Although some said that their physician encouraged them to participate in research studies, others commented that the physician clearance to participation required by some studies was a barrier because it was often difficult to obtain. Participants suggested that all patients (based on characteristics in their electronic medical record) should be automatically invited to participate in a study if they meet the criteria. Lastly, those who had been involved in research wanted to hear the final results of the study, yet most had never received that information from the study team. Almost all OAWCs and caregivers were willing to become team members.

Ability to Make Meaningful Contributions: Few OAWCs and their caregivers had ever heard of the term patient engagement in research, yet most said they would be willing to become a research team member if their voices and contributions could benefit them or future patients/caregivers. They had the time to be involved and said they would want to know in advance the objectives of the project and each meeting. They were willing to be involved for longer periods, although they preferred meeting once monthly or more frequently but for less time. Several OAWCs preferred to work in smaller groups, and preferred having more than $1 \mathrm{OAWC}$ on the team so that they could connect with their peers during the project. Furthermore, having more than 1 OAWC on the team would ensure that continuity of the patient's voice was guaranteed. Notably, some OAWCs pointed out that a potential harm of engaging in research would be the possibility of learning that the prognosis and treatment options of their own disease were not as optimistic as previously believed.

Logistics Required to Facilitate Engagement Amidst Patient Preferences and Healthcare Status: OAWCs and their caregivers raised several logistical issues that needed to be addressed in order to facilitate engagement. Meeting flexibility (ie, day of the week) was important, as were length of the meeting (preferably no more than half a day), timing (not during rush hour and not after dark), providing transportation to those who need it, and accounting for winter conditions. OAWCs and caregivers preferred face-to-face meetings over telephone confer- 
Older Adult Engagement in Research

\begin{tabular}{|c|c|}
\hline Topics Discussed & Findings \\
\hline $\begin{array}{l}\text { Motivation to be on a research } \\
\text { team }\end{array}$ & $\begin{array}{l}\text { - Fewer than half of older adults with cancer had participated in any type of study, caregivers had even less } \\
\text { participation in studies but were very motivated to join a research team } \\
\text { - Most were never invited, others mentioned they were not eligible based on their advanced age } \\
\text { - Some had actively looked for clinical trials and were not eligible } \\
\text { - Patients indicated they don't know how to find clinical trials } \\
\text { - Participants suggested that all patients, based on characteristics in their electronic patient record, should } \\
\text { be invited automatically to participate in a study if they meet the criteria }\end{array}$ \\
\hline $\begin{array}{l}\text { Ability to make meaningful } \\
\text { contributions }\end{array}$ & $\begin{array}{l}\text { - Almost all older adults have never heard of the term patient engagement (research) } \\
\text { - Older adults and their caregivers would be willing to be a research team member if they thought it could } \\
\text { benefit them or future patients/caregivers } \\
\text { - They want to represent the patient's voice } \\
\text { - Potential harm included learning more about own disease (prognosis/treatment) that was not as optimistic } \\
\text { as previously believed } \\
\text { - They would be mostly interested in research on their "own" disease }\end{array}$ \\
\hline $\begin{array}{l}\text { Logistics required to facilitate } \\
\text { engagement amidst patients' } \\
\text { preferences and healthcare status }\end{array}$ & $\begin{array}{l}\text { - Flexibility on days of the week, time, and location (prefer close to home, accessible location, and outside } \\
\text { - Access to computer technology needs to be taken into account (side effects and sensory impairments can } \\
\text { make technology use difficult, as do financial barriers to use technology) } \\
\text { - Transportation (provision if needed) } \\
\text { - Face-to-face meeting is preferred for social contacts; though phone and Skype are useful during bad } \\
\text { weather } \\
\text { - Winter conditions are a barrier to leaving the house in Canada } \\
\text { - Another barrier is not feeling well enough to attend meetings } \\
\text { - Meetings must not be too long and not too tiring, and take into account time for slower pace ("we are a } \\
\text { little slower but we can get there") } \\
\text { - They don't want to be put on the spot and expected to speak but also appreciated the opportunity to } \\
\text { provide input } \\
\text { - Writing/seeing/language barriers } \\
\text { - Divide training into several smaller sessions for ease of retaining information (training over several sessions; } \\
\text { in-person training performed, and if done online it should be supplemented by in-person training) } \\
\text { - Receive materials in advance so that additional information can be read and if necessary search for more } \\
\text { information } \\
\text { - Separate session for questions and answers ahead of the research team meeting and accessibility to a } \\
\text { dedicated researcher they can interact with prior to and after meetings } \\
\text { - Translation of scientific articles and glossary for terminologies } \\
\text { - They prefer "learning as you go" instead of learning in courses }\end{array}$ \\
\hline
\end{tabular}

ences/Skype for social contact and OAWCs preferred locations that were accessible and close to home. The OAWCs and caregivers also thought that researchers should address access and accessibility to computer technology and remember that not all OAWCs will feel well enough to attend all meetings. In addition, the OAWCs who had completed cancer treatment spoke about how fatigue and treatment side effects made using technology more challenging and in-person meetings preferable. Researchers should also be aware of potential language barriers or sensory impairment on the part of OAWCs and/ or their caregivers that could be obstacles to research team participation. Lastly, although OAWCs appreciated the opportunity to provide patient perspective, they did not want to be put on the spot and expected to speak.

OAWCs and caregivers felt that being trained to be a team member (eg, on research conduct and terminology) was important, but that training should be broken up into smaller sessions to facilitate retention of information. They also preferred receiving written training materials before team meetings and having access to a dedicated research team member who could offer a separate question and answer session before meetings. They commented that researchers should provide "translation for scientific articles and a glossary for terminologies." Most participants agreed that it would be a "learn as you go" process, which was preferred over classes and courses in advance of the project.

\section{Discussion}

Our findings show that OAWCs are very interested in participating in studies, especially if they believe it would help them or future patients. Approximately half of the attendees at the public meetings had never been invited to participate in any studies, illustrating older adults' lack of accessibility to research. Although Townsley et $\mathrm{al}^{21}$ found that OAWCs do not seem to actively seek out clinical trials, some in our study said that they had done so but had been told that they were ineligible, whereas others did not 
Puts et al

know where to look. Designing protocols with less restrictive inclusion criteria can help increase older adults' access to studies. ${ }^{5}$ Increased involvement of older adults in the research process itself can help democratize the research process, increase accessibility, and enable their voices to be heard. ${ }^{12,13}$

A recent realist synthesis by $\mathrm{McNeil}$ et $\mathrm{al}^{22}$ developed a 5-stage engagement framework that can be used to enhance engagement. The 5 stages include environment, plan, establish, build, and transition. The environment stage refers to opportunities, or lack thereof, to engage patients and the accessibility issues needed for success, clearly discussed by our participants. The plan stage refers to the investigators planning for engagement; our participants clearly described the logistics that need to be taken into account. The establish stage refers to relationshipbuilding among team members, which links back to the social aspects of being on a team-also clearly described by our participants. The build stage refers to the commitment over time, and the transition stage refers to the end of the research project; our participants clearly indicated they wanted to receive the findings of the projects in which they were engaged. Our findings thus align with this framework ${ }^{22}$ and provide a more detailed insight for engaging OAWCs and their caregivers.

In this study, the top research priorities identified included finding a cure, examining benefits/ harms of complementary and alternative medicine for OAWCs, and improving communication. In Australia, the Cancer Council NSW partnered with Cancer Voices NSW to work with cancer consumers through workshops to set the research agenda for cancer care. ${ }^{23}$ These workshops included 32 participants ranging in age from 18 to 72 years, and top priorities included exploring how to diagnose cancer earlier (before metastatic disease), the extent to which palliative care guidelines are used, nutrition and lifestyle risk factors for cancer, how to address chemotherapy side effects, how to increase cancer screening rates, and what support is needed to help those affected return to normal activities. Some of the priorities align with our identified priorities but some do not, such as cancer screening and use of palliative care guidelines. ${ }^{23}$ This may be due to differences in age of participants (our participants were older and thus some were no longer eligible for the cancer population-based screening programs in Ontario, Canada). Improving communication and finding a cure for cancer were consistently identified as research priorities across all public meetings and focus groups. In a survey study of similar research priorities at The Royal Marsden Hospital, Moorcraft et $\mathrm{al}^{24}$ reported that the priorities varied by age, with younger patients rating early detection of recurrence and palliative treatment higher, and older adults rating side effect management as more important; these were not mentioned by our subjects.

A common challenge cited by researchers in patient engagement studies is logistics of engaging patients, such as funding and time. ${ }^{8}$ In our study, we arranged or compensated for transportation and were able to complete each session on time. Given the importance of engaging patients in research, it is imperative that more funding be allocated for patient engagement initiatives in order to fully support the efforts to actively involve these important partners in research.

Although our findings may not be generalizable, they do provide greater understanding of the importance of including OAWCs on teams, and their training needs to optimize their engagement. Our study included a small sample of OAWCs; it was an exploratory study using qualitative data collection. However, data saturation was obtained for the focus groups. Another limitation was our recruitment of older adults through flyers and newspaper ads. In fact, more than half of the study participants were not currently receiving any cancer treatment. However, these cancer survivors are the most likely to participate in research teams, because many of our OAWCs indicated that they would not have considered participating during their treatments.

Lastly, we did not specify which type of study when discussing OAWC participation on a research team. The study topic would impact their willingness, but because this was a first exploratory study, we chose a broad (any site/stage/treatment) rather than deep (specific site/stage/treatment) approach. Future studies should explore the willingness of older adults to participate on research teams in a larger, more diverse sample as well as explore more in-depth willingness and support needs for different types of studies (eg, clinical trials vs observational studies) and for different clinical groups (eg, early vs late stage, curative vs palliative treatment intent). 
Older Adult Engagement in Research

\section{Conclusions}

Overall, OAWCs are keen to participate in research and to be research team members, yet they are often not invited. Concerted efforts are needed to increase older adults' access to study participation and opportunities to be included in research teams. ${ }^{17,25,26}$ The social aspect of having older adults as part of a research team is important to take into consideration in future endeavors.

Despite the push for increasing engagement of patients and knowledge users, and despite there being a variety of patient engagement theories, frameworks, and models, the best methods for engagement have not been identified, and the impact of engagement on patients and caregivers is not fully understood. $8,11,12,15,22$ Although many studies have examined engagement, most have used qualitative methods, and there is currently no quantitative measurement tool that measures the impact of the engagement on study outcomes. Such a tool is clearly needed, and future similar studies should include one so that different methods of engagement can be compared to develop best practices.

\section{Acknowledgments}

We would like to thank all participants, volunteers, and partners who provided their support for this study: University Health Network, Mount Sinai Hospital, The Scarborough Hospital, St. Michael's Hospital, St. Joseph's Health Centre, Toronto East General Hospital, Sunnybrook Health Sciences Centre, Stronach Regional Cancer Centre, Cancer Care Ontario, The Leukemia \& Lymphoma Society of Canada, Prostate Cancer Canada, Colorectal Cancer Association of Canada, Melanoma Network of Canada, Brain Tumour Foundation of Canada, Lung Cancer Canada, Kidney Cancer Canada, Canadian Cancer Survivor Network, Patients for Patient Safety Canada, CARP (national office and local chapters in Scarborough and Mississauga), Regional Geriatric Program of Toronto, and ELLICSR Health, Wellness \& Cancer Survivorship Centre.

\section{References}

1. Scher KS, Hurria A. Under-representation of older adults in cancer registration trials: known problem, little progress. J Clin Oncol 2012;30:2036-2038

2. Talarico L, Chen G, Pazdur R. Enrollment of elderly patients in clinical trials for cancer drug registration: a 7-year experience by the US Food and Drug Administration. J Clin Oncol 2004;22:4626-4631.

3. Yee KW, Pater JL, Pho L, et al. Enrollment of older patients in cancer treatment trials in Canada: why is age a barrier? J Clin Oncol 2003;21:1618-1623.

4. Al-Refaie WB, Vickers SM, Zhong W, et al. Cancer trials versus the real world in the United States. Ann Surg 2011;254:438-442.

5. Hurria A, Levit LA, Dale W, et al. Improving the evidence base for treating older adults with cancer: American Society of Clinical Oncology statement. J Clin Oncol 2015;33:3826-3833.

6. Al-Refaie WB, Weinberg A, Nelson H. Are older adults adequately represented in surgical oncology trials? Bull Am Coll Surg 2013;98:52-53.

7. Witham MD, George J. Clinical trial design for older people-time for a rethink. QJM 2014;107:15-16.

8. Domecq JP, Prutsky G, Elraiyah T, et al. Patient engagement in research: a systematic review. BMC Health Serv Res 2014;14:89.

9. Woolf SH, Zimmerman E, Haley A, Krist AH. Authentic engagement of patients and communities can transform research, practice, and policy. Health Aff (Millwood) 2016;35:590-594.

10. Dudley L, Gamble C, Preston J, et al. What difference does patient and public involvement make and what are its pathways to impact? Qualitative study of patients and researchers from a cohort of randomised clinical trials. PLoS One 2015;10:e0128817.

11. Carman KL, Workman TA. Engaging patients and consumers in research evidence: applying the conceptual model of patient and family engagement. Patient Educ Couns 2017;100:25-29.

12. Smith SK, Selig W, Harker M, et al. Patient engagement practices in clinical research among patient groups, industry, and academia in the United States: a survey. PLoS One 2015;10:e0140232.

13. Strategy for Patient-Oriented Research-Patient Engagement Framework. Canadian Institutes of Health Research Web stie. Available at: http://www. cihr-irsc.gc.ca/e/48413.html. Accessed August 21, 2017.

14. Welcome to INVOLVE. INVOLVE Web site. Available at: http://www. invo.org.uk/. Accessed August 21, 2017.

15. Forsythe LP, Ellis LE, Edmundson L, et al. Patient and stakeholder engagement in the PCORI pilot projects: description and lessons learned. J Gen Intern Med 2016;31:13-21.

16. PCORI (Patient-Centered Outcomes Research Institute). Engagement Rubric for Applicants. Available at: https://www.pcori.org/sites/default/ files/Engagement-Rubric.pdf. Accessed August 21, 2017.

17. Brett J, Staniszewska S, Mockford C, et al. A systematic review of the impact of patient and public involvement on service users, researchers and communities. Patient 2014;7:387-395.

18. Graneheim UH, Lundman B. Qualitative content analysis in nursing research: concepts, procedures and measures to achieve trustworthiness. Nurse Educ Today 2004;24:105-112.

19. Hsieh HF, Shannon SE. Three approaches to qualitative content analysis. Qual Health Res 2005;15:1277-1288.

20. Elo S, Kyngas H. The qualitative content analysis process. J Adv Nurs 2008;62:107-115.

21. Townsley CA, Chan KK, Pond GR, et al. Understanding the attitudes of the elderly towards enrolment into cancer clinical trials. BMC Cancer 2006;6:34.

22. McNeil H, Elliott J, Huson $\mathrm{K}$, et al. Engaging older adults in health care research and planning: a realist synthesis. Res Involve Engagem 2016;2:10.

23. Saunders C, Crossing S. Towards meeting the research needs of Australian cancer consumers. BMC Res Notes 2012;5:667.

24. Moorcraft SY, Sangha A, Peckitt C, et al. Does cancer research focus on areas of importance to patients? Ecancermedicalscience 2016;10:ed51.

25. Hurria A, Mohile SG, Dale W. Research priorities in geriatric oncology: addressing the needs of an aging population. J Natl Compr Canc Netw 2012;10:286-288.

26. Hurria A, Cohen HJ, Extermann M. Geriatric oncology research in the cooperative groups: a report of a SIOG special meeting. J Geriatr Oncol 2010;1:40-44. 